CERN-PH/TH/2004-054

hep-th/?

\title{
Homogeneous Special Manifolds, Orientifolds and Solvable Coordinates.
}

\author{
R. D'Auria *, S. Ferrara ${ }^{\dagger \sharp}$ and M. Trigiante * \\ $\dagger C E R N$, Theory Division, CH 1211 Geneva 23, Switzerland. \\ $\ddagger I N F N$, Laboratori Nucleari di Frascati, Italy. \\ $\sharp$ University of California, Los Angeles, USA \\ $\star$ Dipartimento di Fisica, Politecnico di Torino \\ C.so Duca degli Abruzzi, 24, I-10129 Torino, and \\ Istituto Nazionale di Fisica Nucleare, Sezione di Torino, \\ Italy
}

\begin{abstract}
We discuss some geometrical properties of the underlying $N=2$ geometry which encompasses some low-energy aspects of $N=1$ orientifolds as well as four dimensional $N=2$ Lagrangians including bulk and open string moduli.

In the former case we illustrate how properly defined involutions allow to define $N=1$ Kähler subspaces of special quaternionic manifolds. In the latter case we show that the full shift symmetry of the brane coordinates, which is abelian in the rigid limit, is partially distorted by bulk fields to a nilpotent algebra.
\end{abstract}




\section{Introduction}

In superstring theory it is possible to construct models where bulk and brane degrees of freedom build a supersymmetric theory in lower dimensions with some number $N$ of supersymmetries left unbroken. Of particular interest are models with $N=4,2,1$ supersymmetry where gauge and matter degrees of freedom are present. Models with $N=4$ supersymmetry are quite restricted. They can be obtained, for example, by compactification of Type IIB theory on a $T^{6}$ orientifold where the bulk sector describes gravity plus six vector multiplets and the brane sector describes some additional gauge multiplets. The Lagrangians of these models (in absence of fluxes) will differ by the way duality symmetries act on the vector fields. Recalling that the $\sigma$-model manifold is

$$
\frac{\mathrm{SU}(1,1)}{\mathrm{U}(1)} \times \frac{\mathrm{SO}(6,6+n)}{\mathrm{SO}(6) \times \mathrm{SO}(6+n)},
$$

the difference come from the way the various vector fields transform under some different subgroups of $\mathrm{SU}(1,1) \times \mathrm{SO}(6,6+n)$. The simplest examples are two extreme cases, the $T^{6} / \mathbb{Z}_{2}$ orientifolds with D3-branes, and Type I superstring, i.e. a Type IIB on a $T^{6}$-orientifold with $D 9$-branes present. In the latter case the full $\mathrm{SO}(6,6+n)$ acts on the $12+n$ vector potentials, while in the former case only a $\mathrm{GL}(6, \mathbb{R}) \times \mathrm{SO}(n)$ acts linearly on the vector potentials. Moreover $\mathrm{SU}(1,1)$ acts linearly on the 12 bulk vectors of the former while it acts as an electric-magnetic duality on the 12 bulk vectors of the latter. A consequence of this situation is that the 15 shift symmetries of the $\mathrm{SO}(6,6) /(\mathrm{SO}(6) \times \mathrm{SO}(6))$ manifold do not act on the 12 bulk vector potentials in the $T^{6} / \mathbb{Z}_{2}$-orientifolds while they act on some potentials in the Type I case.

For $N=2,1$ theories this state of affairs is even more involved because different $D p-$ branes can occur at the same time and so the geometry of bulk and different brane moduli must be explaned. Moreover the compactification manifold can involve curved spaces such as $K 3$ or Calabi-Yau manifolds. In the present paper we consider some geometrical aspects which play a role in the supergravity description of these models.

The paper is organized as follows: in section 2 we give two $N=4$ examples, namely the $T^{6} / \mathbb{Z}_{2}$-orientifold with $D 3$-branes, and Type I on $T^{6}$. We show that the bulk sector of

these theories can be obtained using two different Lie algebra involutions which truncate the original $N=8$ solvable Lie algebra of $\mathrm{E}_{7(7)} / \mathrm{SU}(8)$ to a $\frac{\mathrm{SU}(1,1)}{\mathrm{U}(1)} \times \frac{\mathrm{SO}(6,6)}{\mathrm{SO}(6) \times \mathrm{SO}(6)}$ submanifold.

In section 3 we consider, in the $N=2$ context, a class of homogeneous quaternionic manifolds which allow to obtain, using suitable involutions defined by Cartan isometry generators, different $N=1$ spaces upon truncation. One of these spaces is related to the 
c-map [1, 2] and another to a "dual" Kähler space which occur in Calabi-Yau orientifolds [3, 4, 15, 6]. They differ by the fact that different NS and R-R scalars are present depending on the particular involutions used.

In section 4 we analyze a class of rank 3 homogeneous Very Special Geometries that are relevant for $N=2$ theories in the presence of $D 3$ and $D 7$-branes ${ }^{1}$. We describe the relation between the solvable Lie algebra coordinates and the holomorphic special coordinates and their relation to the brane coordinates as obtained from the combined Born-Infeld, ChernSimons action. We analyze the full set of shift symmetries in the $2 n_{3}+2 n_{7}$ brane coordinates of which only $n_{3}+n_{7}$ are abelian, as in the rigid limit of the Born-Infeld action. The other $n_{3}+n_{7}$ shift symmetries do not commute with the previous ones due to the presence of Chern-Simons terms which must be added to the Born-Infeld action. However the full B$\mathrm{I}+\mathrm{C}-\mathrm{S}$ action is further corrected to reproduce the metric of the predicted Special Geometry. The microscopic origin of these corrections is related to sypersymmetry and gauge anomaly counterterms which arise when compactifying the $D=6$ parent theory down to $D=5$ or $D=4[7,8$.

These results are relevant to understand the relation of these formulations with the computations made in terms of Born-Infeld actions. The relations between different coordinates is also relevant for cosmological applications of supersymmetric models with branes, where shift symmetries in the brane coordinates play an important role [9, 10, 11, 12,

In section 5 we recall the six-dimensional origin of the prepotential and its possible generalizations.

Finally in Appendix $\mathrm{A}$ we recall the geometry of the dual $N=1$ Kähler manifold and give the explicit expression for its Riemann tensor.

\section{$2 \quad N=8$ involutions and two $N=4$ examples}

Let us start reviewing the construction of the two ungauged $N=4$ models describing the bulk sector of Type IIB on $T^{6} / \mathbb{Z}_{2}$ orientifold, in the presence of $D 3$ and Type IIB on $T^{6}$ orientifold in the presence of $D 9$-branes respectively (the latter model, as mentioned in the introduction, being equivalent to the bulk sector of Type I on $T^{6}$ ). The first case was analyzed in detail in [13, 14, 15]. In this section only we shall label by $a, b$ the Dirichlet directions and by $i, j$ the Neumann directions of the internal torus. In the presence of

\footnotetext{
${ }^{1}$ The relation between $D 3 / D 7$ Special Geometry and certain homogeneous manifolds was brought to our attention by A. Van Proeyen.
} 
D3-branes transverse to $T^{6}$ all the compact directions will be Dirichlet and the orientifold projection has the form $\Omega I_{6}(-)^{F_{L}}$, where $\Omega$ is the world-sheet parity and $I_{6}$ is the space parity acting on the torus coordinates. The surviving scalar fields (in the bulk sector) are $\phi, g_{a b}, C_{a b c d}, C_{(0)}$ while the vector fields are $C_{a \mu}, B_{a \mu}$. As far as the latter case is concerned, the orientifold projection (consistent with residual $N=4$ in the presence of $D 9$-branes) consists of the world-sheet parity $\Omega$ only. The surviving scalar fields (in the bulk sector) are $\phi, g_{i j}, C_{i j}, C_{\mu \nu}^{*}\left(C_{\mu \nu}^{*}\right.$ being the scalar field dual to the four-dimensional tensor field $\left.C_{\mu \nu}\right)$ while the vector fields are $C_{i \mu}, G_{\mu}^{i}$. These two models can be obtained as truncations of the $N=8$ theory describing the low-energy limit of Type IIB on $T^{6}$ in absence of fluxes. At the supergravity level the orientifold operations indeed are realized by involutions on the solvable algebra generating the $N=8$ scalar manifold $\mathrm{E}_{7(7)} / \mathrm{SU}(8)$ and on the vector fields. Later on we shall briefly review the solvable Lie algebra formalism. Consider the scaling symmetry defined by a Cartan generator $h$ with respect to which the scalar fields transform with an integer grading $k: \Phi_{k} \rightarrow e^{k \lambda} \Phi_{k}$. This is still a symmetry of the Lagrangian even if we extend the scaling parameter to the complex plane and set $\lambda=i \pi$. This scaling transformation now takes the form:

$$
\Phi_{k} \rightarrow(-)^{k} \Phi_{k} \Rightarrow\left\{\begin{array}{l}
\Phi_{2 \ell} \rightarrow \Phi_{2 \ell} \\
\Phi_{2 \ell+1} \rightarrow-\Phi_{2 \ell+1}
\end{array} .\right.
$$

This implies that setting $\Phi_{2 \ell+1}=0$ defines a consistent truncation of the scalar sector since the corresponding solvable Lie algebra closes. This truncation can be generalized to 4-dimensional $p$-forms by setting to zero all the fields which are odd with respect to the involution $(-)^{h+p}$. As a consequence of this the scalar fields and rank 2 tensor fields of the truncated theory should have even grading with respect to $h$ while vector fields should have an odd grading. This recipe for truncation can be extended to four dimensional gauged supergravities corresponding to string theory compactifications in the presence of fluxes. From the supergravity point of view a flux in the microscopic setting is described by an embedding matrix which is a tensor transforming covariantly with respect to the global symmetry group $\mathscr{G}_{e}$ of the ungauged lagrangian and which defines how the gauge group $\mathscr{G}$ is embedded inside $\mathscr{G}_{e}$. The gauged Lagrangian is still globally invariant under $\mathscr{G}_{e}$ provided that besides the fields also fluxes are transformed. In particular also fluxes, in the lowenergy description, have a well defined grading under any dilation symmetry of the ungauged Lagrangian. Since fluxes are 0-form field strengths, from the four dimensional point of view they can be associated with $p=-1$ forms and therefore our recipe for truncation would require restricting to those fluxes which are even with respect to $(-1)^{h-1}$. This is consistent with the requirement that the minimal couplings $($ Flux $) \times A_{\mu} \times \partial^{\mu} \Phi$ and the non abelian terms $($ Flux $) \times A_{\mu} \times A_{\nu} \times F^{\mu \nu}$ be even under $(-1)^{h}$. Summarizing: in a gauged 
supergravity describing the low-energy limit of a superstring compactification with fluxes, a Cartan generator $h$ of a symmetry of the Lagrangian such that $(-)^{2 h}=+1$ on the bosonic sector, defines a consistent truncation of the theory if we restrict the fields to the following gradings:

$$
\text { truncation in 4-D : }\left\{\begin{array}{l}
\text { scalars }(-1)^{h}=+1 \\
\text { vectors }(-1)^{h}=-1 \\
2 \text {-forms }(-1)^{h}=+1 \\
\text { fluxes/embedding-matrix }(-1)^{h}=-1
\end{array} .\right.
$$

Let us now briefly recall the main facts about the solvable Lie algebra description of homogeneous scalar manifolds in supergravity. We refer the reader to [16, 17] where the relevant conventions are fixed. This representation consists in describing a homogeneous scalar manifold $\mathcal{M}$ as a Lie group generated by a solvable Lie algebra $\operatorname{Solv}(\mathcal{M})$, whose parameters are the scalar fields of the theory:

$$
\mathcal{M}=\exp [\operatorname{Solv}(\mathcal{M})]
$$

All homogeneous manifolds which are relevant to supergravity admit such representation. In the $N=8$ model derived from Type IIB toroidal compactification, the solvable Lie algebra generating the scalar manifold can be described as follows:

$$
\begin{aligned}
\text { Solv }_{7} & =\operatorname{Solv}\left(\frac{\mathrm{E}_{7(7)}}{\mathrm{SU}(8)}\right)=\text { Solv }_{2}+\operatorname{Solv}_{6}+(\mathbf{2}, \mathbf{1 5})_{+1}+\left(\mathbf{1}, \mathbf{1 5}^{\prime}\right)_{+2}+(\mathbf{2}, \mathbf{1})_{+3}, \\
\operatorname{Solv}_{2} & =\operatorname{Solv}\left(\frac{\mathrm{SL}(2, \mathbb{R})_{\mathrm{IIB}}}{\mathrm{SO}(2)}\right)=\left\{\phi H^{\prime}+C_{(0)} t\right\} \\
\text { Solv }_{6} & =\operatorname{Solv}\left(\frac{\mathrm{GL}(6, \mathbb{R})}{\mathrm{SO}(6)}\right)=\left\{\sum_{\Lambda=1}^{6} \log \left(g_{\Lambda \Lambda}\right) H_{\Lambda}+\sum_{\Lambda \neq \Sigma} g_{\Lambda \Sigma} t^{\Lambda}\right\} \\
(\mathbf{2}, \mathbf{1 5})_{+1} & =\left\{B_{\Lambda \Sigma \tau} t^{\Lambda \Sigma \tau}\right\} ;\left(\mathbf{1}, \mathbf{1 5}^{\prime}\right)_{+2}=\left\{\epsilon^{\Lambda_{1} \ldots \Lambda_{6}} C_{\Lambda_{1} \ldots \Lambda_{4}} t_{\Lambda_{5} \Lambda_{6}}\right\}, \\
(\mathbf{2}, \mathbf{1})_{+3} & =\left\{D_{\tau} t^{\tau}\right\}
\end{aligned}
$$

where $\Lambda, \Sigma=1, \ldots, 6$ label the directions of the internal torus, $\mathrm{SL}(2, \mathbb{R})_{\mathrm{IIB}}$ is the duality group of the ten dimensional Type IIB theory, $\tau, \sigma=1,2$ are indices of the doublet of $\mathrm{SL}(2, \mathbb{R})_{\text {IIB }}$ so that $\left\{B_{\Lambda \Sigma \tau}\right\}=\left\{C_{\Lambda \Sigma}, B_{\Lambda \Sigma}\right\}$ and $\left\{D_{\tau}\right\}=\left\{B_{\mu \nu}^{*}, C_{\mu \nu}^{*}\right\}$. The group $\mathrm{GL}(6, \mathbb{R})$ acts transitively on the $T^{6}$ metric moduli $g_{\Lambda \Sigma}$ and the representations in (2.4) refer to the group $\operatorname{SL}(2, \mathbb{R})_{\text {IIB }} \times \operatorname{GL}(6, \mathbb{R})$. Generators denoted by $t$ with various indices are nilpotent while $\left\{H^{\prime}, H_{\Lambda}\right\}$ are the diagonal (non-compact) Cartan generators. The part of the algebraic structure of $\mathrm{Solv}_{7}$ which is not straightforward to deduce consists in the non-vanishing 


\begin{tabular}{|c|c|c|}
\hline field & $(-1)^{H+q}$ & $(-1)^{H^{\prime}+q}$ \\
\hline$\phi$ & + & + \\
$g_{\Lambda \Sigma}$ & + & + \\
$C_{(0)}$ & - & + \\
$B_{\Lambda \Sigma}$ & - & - \\
$C_{\Lambda \Sigma}$ & + & - \\
$C_{\Lambda \Sigma \Gamma \Delta}$ & - & + \\
$B_{\mu \nu}^{*}$ & - & - \\
$C_{\mu \nu}^{*}$ & + & - \\
$G_{\mu}^{\Lambda}$ & + & - \\
$B_{\Lambda \mu}$ & - & + \\
$C_{\Lambda \mu}$ & + & + \\
$C_{\Lambda \Sigma \Gamma \mu}$ & - & - \\
\hline
\end{tabular}

Table 1: two orientifold involutions on the $N=8$ bosonic sector.

commutation relations between the generators $\left\{t^{\Lambda \Sigma \tau}, t_{\Lambda \Sigma}, t^{\tau}\right\}$ :

$$
\begin{aligned}
{\left[t^{\Lambda \Sigma \tau}, t^{\Gamma \Omega \sigma}\right] } & =\frac{1}{2} \varepsilon^{\tau \sigma} \varepsilon^{\Lambda \Sigma \Gamma \Omega \Pi \Delta} t_{\Pi \Delta}, \\
{\left[t_{\Lambda \Sigma}, t^{\Gamma \Omega \tau}\right] } & =2 \delta_{\Lambda \Sigma}^{\Gamma \Omega} t^{\tau} .
\end{aligned}
$$

In order to define the involutions which give rise, upon truncation, to the two $N=4$ models discussed above, let us list in Table 1 the bosonic q-form fields of the $N=8$ model with the gradings with respect to $(-1)^{H+q}$ and $(-1)^{H^{\prime}+q}$ where the two Cartan generators $H$ and $H^{\prime}$ are defined by the property that $C_{\mu \nu}^{*}$ be the scalar with the highest $H$-grading equal to 2 and $C_{(0)}$ be the scalar with the highest $H^{\prime}$-grading equal to 2 (in other words they are the Cartan generators corresponding to the positive roots which define the shift generators of $C_{\mu \nu}^{*}$ and $\left.C_{(0)}\right)$. Recall that in our conventions the Kaluza-Klein vectors are denoted by $G_{\mu}^{\Lambda}=g^{\Lambda \Sigma} g_{\Sigma \mu}$. If we restrict to fields with positive grading with respect to $(-1)^{H+q}$ we obtain the bosonic part of the bulk sector of Type I theory on $T^{6}$ while restricting to those with positive grading with respect to $(-1)^{H^{\prime}+q}$ the resulting fields fit the bulk sector of Type IIB theory on $T^{6} / \mathbb{Z}_{2}$ orientifold. In [17] the gauged $N=8$ supergravity describing the low-energy limit of Type IIB on $T^{6}$ with fluxes was constructed and it was shown how the various $N=4$ gauged models constructed in [18, 19] and describing Type IIB on $T^{p-3} \times T^{9-p} / \mathbb{Z}_{2}$ orientifolds in the presence of $D p$-branes and fluxes, could be obtained as consistent truncations of the former model (the $p=3$ and $p=9$ cases correspond to the two $N=4$ models that we are discussing in some detail in this section). These truncations in the various cases can be associated, through the recipe discussed above, to the Cartan generator $H_{p-3}$ corresponding 
to the positive root which defines the axion $C_{(p-3)}$, with components along the Neumann directions $T^{p-3}$. With respect $(-1)^{H_{p-3}}$, for instance, a $\mathrm{R}-\mathrm{R} q+1$-form field strength and the Kalb-Ramond field strengths have grading:

$$
\begin{aligned}
& F_{\mu_{1} \ldots \mu_{q+1} i_{1} \ldots i_{k_{+}} a_{1} \ldots a_{k_{-}}} \leftrightarrow(-1)^{H_{p-3}}=(-1)^{\frac{1}{2}\left(7-p+k_{+}-k_{-}-q\right)}, \\
& \mathscr{H}_{\mu_{1} \ldots \mu_{q+1} i_{1} \ldots i_{k_{+}} a_{1} \ldots a_{k_{-}}} \leftrightarrow(-1)^{H_{p-3}}=(-1)^{\frac{1}{2}\left(k_{+}-k_{-}-q\right)} ; k_{+}+k_{-}+q=2,
\end{aligned}
$$

where $k_{+}, k_{-}$are the number of indices along the Neumann and Dirichlet directions of the torus respectively. As for the metric and Kaluza-Klein vectors, $g_{i j}, g_{a b}$ have $(-1)^{H_{p-3}-}$ grading,$+ g_{i a}$ have grading -,$G_{\mu}^{i}$ grading - and $G_{\mu}^{a}$ grading + .

Below we give, for completeness, the kinetic matrix $\mathscr{N}$ of the vector fields in the two $N=4$ models, including the respective boundary degrees of freedom. The kinetic terms for the vector fields have the general form:

$$
\operatorname{Im} \mathscr{N}_{N M} F_{\mu \nu}^{N} F^{M \mu \nu}+\frac{1}{2} \operatorname{Re} \mathscr{N}_{N M} \epsilon^{\mu \nu \rho \sigma} F_{\mu \nu}^{N} F_{\rho \sigma}^{M}
$$

where $N, M$ run over the total number of vector fields. Let us consider first the case of Type IIB on $T^{6}$ orientifold in the presence of $D 9$-branes. We denote by $\mathscr{F}^{i}, F_{i}, F^{v}$ the field strengths of $G_{\mu}^{i}, C_{i \mu}, A_{\mu}^{v}$ respectively (where $v$ in this section only runs over the number $n_{9}$ of $D 9$-branes, $A_{\mu}^{v}$ and $a_{i}^{v}$ are the space-time and $T^{6}$ components of the gauge vectors on the boundary theory respectively). From the index structure of the electric-magnetic field strengths $\left(\mathscr{F}^{i}, F_{i}, F^{v}, \tilde{\mathscr{F}}_{i}, \tilde{F}^{i}, \tilde{F}_{v}\right)$ we readily see that the whole group $\mathrm{SO}\left(6,6+n_{9}\right)$ is a global symmetry of the Lagrangian since it has a duality action which is block diagonal, namely has a separate linear action on the electric and magnetic components. On the other hand the $\mathrm{SL}(2, \mathbb{R})$ factor in the duality group, which acts transitively on the scalars $\left\{V_{6} e^{\frac{\phi}{2}}, C_{\mu \nu}^{*}\right\}\left(V_{6}\right.$ being the volume of $\left.T^{6}\right)$, is non perturbative since $\left(\mathscr{F}^{i}, \tilde{F}^{i}\right) \in\left(\mathbf{2}, \mathbf{6}^{\prime}\right)_{+1}$ and $\left(F_{i}, \tilde{\mathscr{F}}_{i}\right) \in(\mathbf{2}, \mathbf{6})_{-1}$ with respect to $\operatorname{SL}(2, \mathbb{R}) \times \operatorname{GL}(6, \mathbb{R})$. This group therefore is not a symmetry of the Lagrangian. The matrix $\mathscr{N}_{M N}$ is:

$$
\begin{aligned}
\mathscr{N} & =-c \eta-\mathrm{i} e^{\phi} L^{-1 T} L^{-1}=\left(\begin{array}{ccc}
\mathscr{N}_{i j} & \mathscr{N}_{i}^{j} & \mathscr{N}_{i}^{v} \\
* & \mathscr{N}^{i j} & \mathscr{N}^{i v} \\
* & * & \mathscr{N}^{u v}
\end{array}\right) \\
\mathscr{N}_{i j} & =-\mathrm{i} e^{\phi}\left(E_{i}^{\hat{k}} E_{j}^{\hat{k}}+\tilde{C}_{i k} \tilde{C}_{j \ell} E^{-1 k}{ }_{\hat{k}} E^{-1 \ell}{ }_{\hat{k}}+a_{i}^{v} a_{j}^{v}\right) \\
\mathscr{N}_{i}^{j} & =-c \delta_{i}^{j}-\mathrm{i} e^{\phi} \tilde{C}_{i k} E^{-1 k}{ }_{\hat{k}} E^{-1 j} \hat{k} \\
\mathscr{N}_{i}^{v} & =\mathrm{i} e^{\phi}\left(\tilde{C}_{i k} E^{-1 k}{ }_{\hat{k}} E^{-1 j}{ }_{\hat{k}}^{v} a_{j}^{v}+a_{i}^{v}\right) \\
\mathscr{N}^{i j} & =-\mathrm{i} e^{\phi} E^{-1 i}{ }_{\hat{k}} E^{-1 j}{ }_{\hat{k}} \\
\mathscr{N}^{i v} & =\mathrm{i} e^{\phi} E_{\hat{k}}^{-1 i} E_{\hat{k}}^{-1 j} a_{j}^{v}
\end{aligned}
$$




$$
\begin{aligned}
\mathscr{N}^{u v} & =c \delta^{u v}-\mathrm{i} e^{\phi}\left(\delta^{u v}+a_{i}^{u} a_{j}^{v} E_{\hat{k}}^{-1 i} E_{\hat{k}}^{-1 j}\right) \\
\tilde{C}_{i j} & =C_{i j}+\frac{1}{2} a_{i}^{v} a_{j}^{v} ; c \equiv C_{\mu \nu}^{*} ; \eta_{M N}=\left(\begin{array}{ccc}
0 & \mathbb{1}_{6 \times 6} & 0 \\
\mathbb{1}_{6 \times 6} & 0 & 0 \\
0 & 0 & -\mathbb{1}_{n_{9} \times n_{9}}
\end{array}\right)
\end{aligned}
$$

where $L$ denotes the coset representative of $\mathrm{SO}\left(6,6+n_{9}\right) /\left[\mathrm{SO}(6) \times \mathrm{SO}\left(6+n_{9}\right)\right]$ and $E$ the coset representative of $\mathrm{GL}(6, \mathbb{R}) / \mathrm{SO}(6)$.

Let us now consider the $T^{6} / \mathbb{Z}_{2}$ orientifold model in the presence of $n_{3} D 3$-branes and denote by $F_{a \tau}, F^{v}$ the field strengths of $B_{\mu a \tau}, A_{\mu}^{r}$ respectively (where $r$ runs over the number $n_{3}$ of $D 3$-branes, $A_{\mu}^{r}$ are the vectors on the boundary theory and $a^{a r}$ the $T^{6}$ coordinates of the $r^{\text {th }} D 3$-brane). As it is apparent from the index structure of the electric-magnetic field strengths with respect to $\mathrm{SL}(2, \mathbb{R}) \times \mathrm{GL}(6, \mathbb{R}) \times \mathrm{SO}\left(n_{3}\right)$ only the subalgebra $g l(6, \mathbb{R})+$ $s o\left(n_{3}\right)+\left(\mathbf{1 5}^{\prime}, \mathbf{1}\right)_{+2}+\left(\mathbf{6}^{\prime}, \mathbf{1}\right)_{+1}$ generates the global symmetry group of the Lagrangian. The components of the matrix $\mathscr{N}$ are:

$$
\begin{aligned}
\mathscr{N}^{(1 a)(1 b)} & =-i e^{-\phi}\left(E^{-1} E^{-1 T}\right)^{a b} \\
\mathscr{N}^{(2 a)(1 b)} & =i C_{(0)} e^{-\phi}\left(E^{-1} E^{-1 T}\right)^{a b}-\tilde{C}^{a b} \\
\mathscr{N}^{(2 a)(2 b)} & =-i\left[e^{\phi}\left(E^{-1} E^{-1 T}+a a^{T}\right)^{a b}+e^{-\phi} C_{(0)}^{2}\left(E^{-1} E^{-1 T}\right)^{a b}\right]-C_{(0)}\left(a a^{T}\right)^{a b} \\
\mathscr{N}^{(1 a) r} & =-a^{a r} \\
\mathscr{N}^{(2 a) r} & =a^{a r}\left(C_{(0)}+i e^{\phi}\right) \\
\mathscr{N}^{r s} & =-\delta^{r s}\left(C_{(0)}+i e^{\phi}\right) \\
\tilde{C}^{a b} & =B^{a b}-\frac{1}{2}\left(a a^{T}\right)^{a b}
\end{aligned}
$$

where $B^{a b}$ are related to $C_{a b c d}$ by duality on the internal torus.

\section{Alekseevski structure of a homogeneous quaternionic algebra}

In this section we shall briefly review the structure of the solvable Lie algebra generating certain homogeneous quaternionic manifolds $\mathcal{M}_{Q}$ (quaternionic algebra) [20, 21, 22, [23. This will be useful in order

i) to define, using involutions of the algebra, truncations of the manifold corresponding for instance to the $N=2$ "c-dual" Special Kähler manifold or to the $N=1$ Kähler manifold arising from Calabi-Yau orientifold compactifications [3, 4, 5, 6] of Type IIB theory; 
ii) to study the relations between the geometrical properties of these truncations and in particular to show that for symmetric manifolds the $N=2$ Special Kähler and the dual $N=1$ Kähler manifolds are of the same kind.

We shall restrict ourselves to "Very Special" homogeneous quaternionic manifolds of rank 4. Let the quaternionic dimension of $\mathcal{M}_{Q}$ be $n+1$. The corresponding quaternionic algebra $V$ has the form:

$$
\begin{aligned}
\mathcal{M}_{Q} & =\exp (V) ; V=U+\tilde{U} \\
{[U, U] } & =U ;[U, \tilde{U}]=\tilde{U} ;[\tilde{U}, \tilde{U}]=U
\end{aligned}
$$

where $U$ is an algebra generating a Kähler submanifold and is stable with respect to the action of a complex structure $J_{1}: J_{1} U=U$. $\tilde{U}$ is related to $U$ by the action of a second complex structure $J_{2}: \tilde{U}=J_{2} U$, which, together with $J_{1}$ and $J_{3}=J_{1} J_{2}$ generates the quaternionic structure of $\mathcal{M}_{Q}$. $U$ has a linear adjoint action on the space $\tilde{U}$ which is symplectic with respect to a suitable form $\hat{J}$ expressed in terms of $J_{1}$. The structure of $U$ can be represented as follows:

$$
\begin{aligned}
U & =F_{0}+F_{1}+F_{2}+F_{3}+X^{ \pm}+Y^{ \pm}+Z^{ \pm} \\
{\left[F_{I}, F_{J}\right] } & =0 \quad I, J=0,1,2,3 \\
F_{I} & =\left\{h_{I}, g_{I}\right\} ; \quad\left[h_{I}, g_{I}\right]=g_{I}
\end{aligned}
$$

the solvable subalgebras $F_{I}$ generate four $\mathrm{SL}(2, \mathbb{R}) / \mathrm{SO}(2)$ submanifolds of $\mathcal{M}_{Q}$. The generators $h_{I}$ define the Cartan subalgebra of $V$. The six spaces $X^{ \pm}, Y^{ \pm}, Z^{ \pm}$consist of nilpotent generators and $\operatorname{dim} X^{ \pm}+\operatorname{dim} Y^{ \pm}+\operatorname{dim} Z^{ \pm}=n-3$.

We shall in general denote by $W(S o l v)$ the normal Kähler manifold generated by the solvable Lie algebra Solv. In particular $W\left(F_{1}+F_{2}+F_{3}+X^{ \pm}+Y^{ \pm}+Z^{ \pm}\right)$is the Special Kähler manifold which corresponds to $\mathcal{M}_{Q}$ through the c-map.

The generators of $\tilde{U}$, denoted by $\left\{p^{I}, q_{I}, \tilde{X}^{ \pm}, \tilde{Y}^{ \pm}, \tilde{Z}^{ \pm}\right\}$can be arranged in a symplectic vector with respect to the adjoint action of $U$ :

$$
\left(\begin{array}{c}
v^{\lambda} \\
w_{\sigma}
\end{array}\right) ; v^{\lambda}=\left(\begin{array}{c}
p_{0} \\
p_{\alpha} \\
\tilde{X}^{-} \\
\tilde{Y}^{-} \\
\tilde{Z}^{-}
\end{array}\right) ; w_{\sigma}=\left(\begin{array}{c}
q_{0} \\
q_{\alpha} \\
\tilde{X}^{+} \\
\tilde{Y}^{+} \\
\tilde{Z}^{+}
\end{array}\right)
$$

The algebraic structure of the whole algebra $V$ can be deduced from the gradings of the various nilpotent generators with respect to $h_{I}$ which are listed in Table 2 . In this list we 


\begin{tabular}{|c|c|c|c|c|}
\hline gen. & field & grading & H-grading & $\mathrm{H}^{\prime}$-grading \\
\hline$g_{0}$ & $B_{\mu \nu}^{*}$ & $(1,0,0,0)$ & 1 & 1 \\
\hline$g_{1}$ & $B_{(2)}$ & $(0,1,0,0)$ & 1 & -1 \\
\hline$g_{2}$ & $B_{(2)}$ & $(0,0,1,0)$ & 1 & -1 \\
\hline$g_{3}$ & $B_{(2)}$ & $(0,0,0,1)$ & 1 & -1 \\
\hline$X^{ \pm}$ & $\left\{B_{(2)}\right.$ & $\left(0,0, \frac{1}{2}, \pm \frac{1}{2}\right)$ & $\int 1$ & -1 \\
\hline$Y^{ \pm}$ & $\stackrel{g}{B}_{(2)}$ & $\left(0, \frac{1}{2}, 0, \pm \frac{1}{2}\right)$ & \} $\begin{array}{l}0 \\
1\end{array}$ & $\frac{0}{-1}$ \\
\hline$Z^{ \pm}$ & $g_{(2)}$ & $(0 \underline{1}+\underline{1} 0)$ & $\left\{\begin{array}{l}0 \\
1\end{array}\right.$ & $\begin{array}{l}0 \\
-1\end{array}$ \\
\hline & $g$ & $(0, \overline{2}, \pm \overline{2}, 0)$ & 0 & 0 \\
\hline$p_{0}$ & $C_{\mu \nu}^{*}$ & $\left(\frac{1}{2}, \frac{1}{2}, \frac{1}{2}, \frac{1}{2}\right)$ & 2 & -1 \\
\hline$q_{0}$ & $C_{(0)}$ & $\left(\frac{1}{2},-\frac{1}{2},-\frac{1}{2},-\frac{1}{2}\right)$ & -1 & 2 \\
\hline$p_{1}$ & $C_{(2)}$ & $\left(\frac{1}{2}, \frac{1}{2},-\frac{1}{2},-\frac{1}{2}\right)$ & 0 & 1 \\
\hline$q_{1}$ & $C_{(4)}$ & $\left(\frac{1}{2},-\frac{1}{2}, \frac{1}{2}, \frac{1}{2}\right)$ & 1 & 0 \\
\hline$p_{2}$ & $C_{(2)}$ & $\left(\frac{1}{2},-\frac{1}{2}, \frac{1}{2},-\frac{1}{2}\right)$ & 0 & 1 \\
\hline$q_{2}$ & $C_{(4)}$ & $\left(\frac{1}{2}, \frac{1}{2},-\frac{1}{2}, \frac{1}{2}\right)$ & 1 & 0 \\
\hline$p_{3}$ & $C_{(2)}$ & $\left(\frac{1}{2},-\frac{1}{2},-\frac{1}{2}, \frac{1}{2}\right)$ & 0 & 1 \\
\hline$q_{3}$ & $C_{(4)}$ & $\left(\frac{1}{2}, \frac{1}{2}, \frac{1}{2},-\frac{1}{2}\right)$ & 1 & 0 \\
\hline$\tilde{X}^{ \pm}$ & $\left\{C_{(4)}\right.$ & $\left(\frac{1}{2}, \pm \frac{1}{2}, 0,0\right)$ & $(1$ & 0 \\
\hline$\tilde{\tilde{u}}$ & $\begin{array}{l}C_{(2)} \\
C_{(4)}\end{array}$ & & $\begin{array}{l}0 \\
1\end{array}$ & $\begin{array}{l}1 \\
0\end{array}$ \\
\hline$Y^{ \pm}$ & $C_{(2)}$ & $\left(\frac{1}{2}, 0, \pm \frac{1}{2}, 0\right)$ & 0 & 1 \\
\hline$\tilde{Z}^{ \pm}$ & $C_{(4)}$ & $\left(\frac{1}{a}, 0,0, \pm \frac{1}{a}\right)$ & 1 & 0 \\
\hline & $C_{(2)}$ & $+\overline{2})$ & 0 & 1 \\
\hline
\end{tabular}

Table 2: generators of $V$ and their gradings. 
have also specified for each nilpotent generator the scalar field which parametrizes it in terms of the corresponding ten-dimensional parent fields: $g$ represents the metric, $B_{(2)}, C_{(2)}, C_{(4)}$ the Type IIB forms. This generator-field correspondence can be justified as follows. We start from knowing that $U$ is parametrized by NS-NS fields coming either from $B_{(2)}$ or from the metric moduli $g$ and the dilaton $\phi$. The generators related to $B_{(2)}\left(T^{B}\right)$ can be identified form the typical commutation property with those corresponding to metric moduli $\left(T^{g}\right)$ or dilaton $\left(T^{\phi}=h_{0}\right)$

$$
\left[T^{B}, T^{g}\right]=T^{B} ;,\left[T^{B}, T^{\phi}\right]=T^{B}
$$

Thus we have that

$$
\begin{aligned}
\left\{T^{B}\right\} & =\left\{g_{0}, g_{\alpha}, X^{+}, Y^{+}, Z^{+}\right\} \\
\left\{T^{\phi}, T^{g}\right\} & =\left\{h_{0}, h_{\alpha}, X^{-}, Y^{-}, Z^{-}\right\}
\end{aligned}
$$

If we denote by the generic symbols $T^{C_{0}}, T^{C_{2}}, T^{C_{2}^{*}}, T^{C_{4}}$ the nilpotent generators parametrizing the $\mathrm{R}-\mathrm{R}$ scalars coming from $C_{(0)}, C_{(2)}, C_{\mu \nu}^{*}, C_{(4)}$, the following general commutation properties can help us defining the (qualitative) correspondence:

$$
\begin{aligned}
{\left[T^{B}, T^{C_{0}}\right] } & =T^{C_{2}} ;\left[T^{B}, T^{C_{2}}\right]=T^{C_{4}} ;\left[T^{B}, T^{C_{4}}\right]=T^{C_{2}^{*}} \\
{\left[T^{C_{2}}, T^{C_{4}}\right] } & =\left\{g_{0}\right\} ;\left[T^{C_{0}}, T^{C_{2}^{*}}\right]=\left\{g_{0}\right\} ;
\end{aligned}
$$

To make contact with the notation used in the literature (see [22, 23, 6]) for the coordinates and solvable isometries of the quaternionic manifold let us specify their correspondence with the solvable generators used here:

$$
\begin{aligned}
\left\{h_{0}, g_{0}, q_{0}, p_{0}\right\} & \leftrightarrow\left\{D, \tilde{\Phi}, \zeta^{0}, \tilde{\zeta}_{0}\right\} \\
\left\{q_{\alpha}, \tilde{X}^{+}, \tilde{Y}^{+}, \tilde{Z}^{+}\right\} & \leftrightarrow\left\{\tilde{\zeta}_{a}\right\} \\
\left\{p_{\alpha}, \tilde{X}^{-}, \tilde{Y}^{-}, \tilde{Z}^{-}\right\} & \leftrightarrow\left\{\zeta^{a}\right\} \\
\left\{h_{\alpha}, X^{-}, Y^{-}, Z^{-}\right\} & \leftrightarrow\left\{y^{a}\right\} \\
\left\{g_{\alpha}, X^{+}, Y^{+}, Z^{+}\right\} & \leftrightarrow\left\{x^{a}\right\} \\
\left\{q_{\alpha}, \tilde{X}^{+}, \tilde{Y}^{+}, \tilde{Z}^{+}\right\} & =\left\{\beta_{a}\right\} ;\left\{p_{\alpha}, \tilde{X}^{-}, \tilde{Y}^{-}, \tilde{Z}^{-}\right\}=\left\{\alpha^{a}\right\} \\
q_{0} & =\alpha^{0} ; p_{0}=\beta_{0}
\end{aligned}
$$

As far as the $X, Y, Z$ generators are concerned the quaternionic structure of the manifold requires that either $\operatorname{dim}\left(X^{ \pm}\right)=0 \bmod 4$ or if $\operatorname{dim}\left(X^{ \pm}\right) \neq 0 \bmod 4$ then we should have 
$\operatorname{dim}\left(Y^{ \pm}\right)=\operatorname{dim}\left(Z^{ \pm}\right)$[22, 23]. From the grading structure we also deduce that $\left[X^{-}, Z^{-}\right]=$ $Y^{-}$.

As far as the $\operatorname{dim}\left(X^{ \pm}\right)=0$ case is concerned, if $p=\operatorname{dim}\left(Y^{ \pm}\right)$and $q=\operatorname{dim}\left(Z^{ \pm}\right)$the Kähler algebra is denoted by $K(p, q)$ and the quaternionic algebra by $W(p, q)$.

In the $D 3 / D 7$ problem the scalar manifold of the vector multiplet sector is generated by an algebra $K(p, q)$, where $p=n_{3}$ and $q=n_{7}$. We shall come back to these manifolds in the next section.

Scaling symmetries and truncations Let us consider three relevant truncations of the scalar sector defined by three different Cartan generators, according to the recipe given in section 2

If we consider $h=2 h_{0}$ we see that all the $\mathrm{R}-\mathrm{R}$ generators are odd and the truncation leaves the generators $h_{0}, g_{0}$ and the Kähler algebra obtained under c-map:

$$
\begin{aligned}
W\left(h_{0}, g_{0}\right) & \times W \\
W & =W\left(g_{\alpha}, h_{\alpha}, X^{ \pm}, Y^{ \pm}, Z^{ \pm}\right)
\end{aligned}
$$

If we consider $h=H=h_{0}+h_{1}+h_{2}+h_{3}$, from the table above we deduce that the truncation leaves:

$$
\begin{aligned}
W\left(h_{0}^{\prime}, p_{0}\right) & \times W_{1} \\
W_{1} & =W\left(h_{\alpha}^{\prime}, X^{-}, Y^{-}, Z^{-}, p_{\alpha}, \tilde{X}^{-}, \tilde{Y}^{-}, \tilde{Z}^{-}\right)
\end{aligned}
$$

corresponding to the fields:

$$
\left\{\phi, G, C_{\mu \nu}^{*}, C_{(2)}\right\}
$$

where $h_{I}^{\prime}$ are suitable linear combinations of $h_{I}$. The manifold $W_{1}$ is still Special Kähler and defines the vector multiplet sector of the sigma model obtained by reducing Type I theory on a Calabi-Yau manifold.

For each nilpotent isometry generator $A$ we may formally consider its negative $\hat{A}$ defined

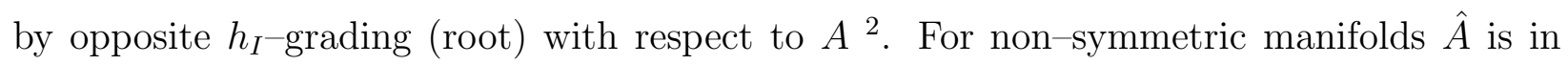

\footnotetext{
${ }^{2}$ Notice that in the literature (see for instance 22, 23, 6]) the hatted symbols are used to denote generators which have opposite $h_{0}$ grading but same $h_{\alpha}$ grading with respect to the positive counterparts (in the nonhomogeneous cases $h_{\alpha}$ do not always exist). Here for convenience we used this notation for generators with opposite $h_{0}$ and $h_{\alpha}$-grading. Therefore the nilpotent generators of $\operatorname{SL}(2, \mathbb{R})_{\text {IIB }}$ are in the two notations: $q_{0}=\alpha^{0}, \hat{q}_{0}=\hat{\beta}_{0}$.
} 
general not an isometry. If $\hat{A}$ is an isometry, then we can define the Weyl transformation $\mathcal{O}_{A}=\exp \left(\frac{\pi}{2}(A-\hat{A})\right)$ which, besides being an automorphism of the isometry algebra, namely preserving the whole algebraic structure of $V$, maps Cartan generators into Cartan generators. For very special manifolds the generator $\hat{q}_{0}$ is always an isometry since $h_{0}, q_{0}, \hat{q}_{0}$ generate the $\mathrm{SL}(2, \mathbb{R})_{\text {IIB }}$ ten-dimensional type IIB duality and the mapping between $W$ and $W_{1}$ is provided by the discrete Weyl transformation $\mathcal{O}_{q_{0}}$ :

$$
\begin{aligned}
\mathcal{O}_{q_{0}} W\left(h_{0}, g_{0}\right) \mathcal{O}_{q_{0}}^{-1} & =W\left(h_{0}^{\prime}, p_{0}\right) \\
\mathcal{O}_{q_{0}} W \mathcal{O}_{q_{0}}^{-1} & =W_{1}
\end{aligned}
$$

Since the two algebras are mapped into each other by an automorphism of the quaternionic algebra which is also an isometry, and since the geometric properties of these manifolds are encoded the commutation relations between their generators and the remaining generators in $V$, we deduce that the $W_{1}$ should be a special Kähler manifold as well, of the same kind as $W$.

If we consider $h=H^{\prime}=h_{0}-h_{1}-h_{2}-h_{3}$, from Table 2 we deduce that the truncation leaves:

$$
\begin{aligned}
W\left(h_{0}^{\prime \prime}, q_{0}\right) & \times W_{2} \\
W_{2} & =W\left(h_{\alpha}^{\prime \prime}, X^{-}, Y^{-}, Z^{-}, q_{\alpha}, \tilde{X}^{+}, \tilde{Y}^{+}, \tilde{Z}^{+}\right)
\end{aligned}
$$

corresponding to the fields:

$$
\left\{\phi, G, C_{(0)}, C_{(4)}\right\}
$$

The generators $h_{I}^{\prime \prime}$ are related to $h_{I}$ by a linear transformation. The manifold $W_{2}$ defines the sigma model of the $N=1$ theory obtained by reduction of Type IIB supergravity on a Calabi-Yau orientifold. It was constructed in [4, 5] ad it is referred to as "dual" Kähler manifold. The relation between $W$ and $W_{2}$ would involve the action of generators corresponding to negative roots other than $\hat{q}_{0}$ and which are present among the isometries of the manifold only for symmetric manifolds. Let us show this. Suppose first that the special Kähler manifold $W$ is symmetric. In this case, for each nilpotent generator $A$ in its solvable algebra the corresponding negative counterpart $\hat{A}$ is an isometry. In particular the generators $\hat{g}_{\alpha}$ are isometries and so are the Weyl transformations $\mathcal{O}_{g_{\alpha}}$. Let us prove that the following Weyl transformation:

$$
\mathcal{O}=\mathcal{O}_{g_{3}} \mathcal{O}_{g_{2}} \mathcal{O}_{g_{1}} \mathcal{O}_{q_{0}}
$$


which is an isometry, maps the dual Kähler manifolds into each other:

$$
\begin{aligned}
\mathcal{O} W\left(h_{0}, g_{0}\right) \mathcal{O}^{-1} & =W\left(h_{0}^{\prime \prime}, q_{0}\right) \\
\mathcal{O} W \mathcal{O}^{-1} & =W_{2}
\end{aligned}
$$

Indeed by inspection of the various gradings we can show that:

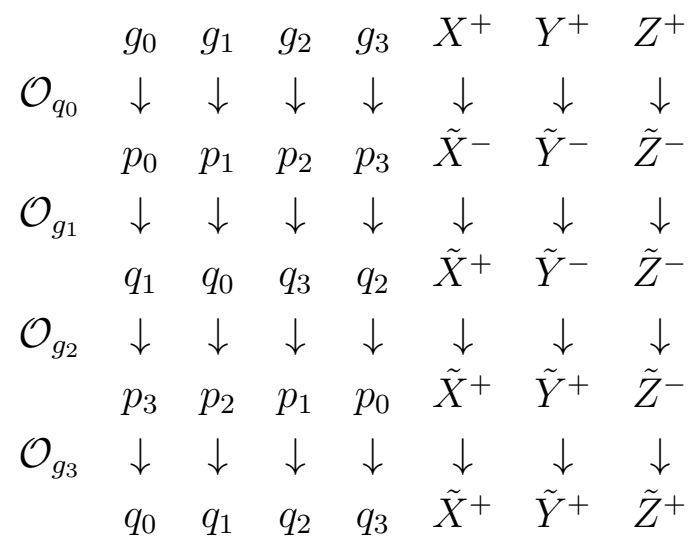

The action of $\mathcal{O}$ on the other generators parametrized by the dilaton and the metric moduli has the following effect:

$$
\mathcal{O}\left\{h_{0}, h_{\alpha}, X^{-}, Y^{-}, Z^{-}\right\} \mathcal{O}^{-1}=\left\{h_{0}^{\prime \prime}, h_{\alpha}^{\prime \prime}, \hat{X}^{-}, \hat{Y}^{-}, \hat{Z}^{-}\right\}
$$

due to the symmetry property of $W$ the generators $\left\{h_{0}, h_{\alpha}, \hat{X}^{-}, \hat{Y}^{-}, \hat{Z}^{-}\right\}$are still in the isometry group of the dual Kähler manifold $W_{2}$. This proves eqs. (3.15) and thus that:

If the original Special Kähler manifold $W$ is symmetric, the dual one $W_{2}$ will be a Special Kähler manifold of the same kind. In Appendix A we shall derive the Riemann tensor for the dual Kähler manifold and show that it coincides which the one of the original Special Kähler manifold in the symmetric case.

Finally it can be shown that if $W$ is symmetric the full quaternionic algebra is symmetric as well. Indeed by successive action of $\hat{g}_{\alpha}$ on $\hat{q}_{0}$ we generate $\hat{p}_{0}$ which is thus also an isometry. The commutator $\left[\hat{q}_{0}, \hat{p}_{0}\right]=\hat{g}_{0}$, denoted in the literature by $\epsilon^{-}$, and all the other negative counterparts of the quaternionic generators can be generated as commutators of isometries, thus belonging to the isometry algebra as well.

Manifolds which are "dual" but not symmetric are in general different. As an example we can consider "dual" Kähler manifolds which are homogeneous but not symmetric. For instance we may choose $W=K(1,1)$ of complex dimension 5 . To show that the two manifolds are different it suffices to show that the corresponding scalar curvatures are different. Indeed one finds:

$$
R(W)=-18 ; \quad R\left(W_{2}\right)=-16 .
$$




\section{Special coordinates, solvable coordinates and B.I. action}

The prepotential for the spacial geometry of the $D 3-D 7$ system is

$$
\mathcal{F}=s t u-\frac{1}{2} s\left(x^{i}\right)^{2}-\frac{1}{2} u\left(y^{r}\right)^{2} .
$$

This prepotential was obtained in [24, by using arguments based on duality symmetry, four dimensional Chern-Simons terms coming from the p-brane couplings as well as couplings of vector multiplets in $D=4$ and $D=8$.

A similar result was advocated in [7, 8] by performing first a $K 3$ reduction to $D=6$ and then further compactifying the theory to $D=4$ on $T^{2}$.

The subtlety of this derivation is that the naive Born-Infeld action derived for D5 and $D 9$ branes in $D=6$ gives kinetic terms for the scalar fields which, at the classical level, are inconsistent with $N=2$ supersymmetry. This is a consequence of the fact that anomalies are present in the theory, as in the $D=10$ case. The mixed anomaly local counterterms are advocated to make the Lagrangian $N=2$ supersymmetric in $D=4$.

Therefore the corrected Lagrangian, in the original brane coordinates is highly nonpolinomial. In fact the original Born-Infeld, Chern-Simons naive (additive) classical scalar action

$$
\begin{aligned}
& \frac{\left|\partial s^{\prime}+c^{r} \partial d^{r}\right|^{2}}{\left(s^{\prime}-\bar{s}^{\prime}\right)^{2}}+\frac{\left|\partial u^{\prime}+a^{i} \partial b^{i}\right|^{2}}{\left(u^{\prime}-\bar{u}^{\prime}\right)^{2}}+\frac{\left|t^{\prime} \partial d^{r}+\partial c^{r}\right|^{2}}{\left(s^{\prime}-\bar{s}^{\prime}\right)\left(t^{\prime}-\bar{t}^{\prime}\right)}+\frac{\left|t^{\prime} \partial b^{i}+\partial a^{i}\right|^{2}}{\left(u^{\prime}-\bar{u}^{\prime}\right)\left(t^{\prime}-\bar{t}^{\prime}\right)}+\frac{\left|\partial t^{\prime}\right|^{2}}{\left(t^{\prime}-\bar{t}^{\prime}\right)^{2}} \\
& s^{\prime}=s-\frac{1}{2} d^{r} y^{r} ; u^{\prime}=u-\frac{1}{2} b^{i} x^{i} ; t^{\prime}=t \\
& x^{i}=a^{i}+t b^{i} ; y^{r}=c^{r}+t d^{r},
\end{aligned}
$$

has a metric which was shown [8] to be Kähler with Kähler potential ${ }^{3}$

$$
\begin{aligned}
K= & -\log \left[(s-\bar{s})(t-\bar{t})-\frac{1}{2}\left(y^{r}-\bar{y}^{r}\right)^{2}\right]-\log \left[(u-\bar{u})(t-\bar{t})-\frac{1}{2}\left(x^{i}-\bar{x}^{i}\right)^{2}\right]+\log (t-\bar{t}) \\
& =-\log Y_{S K}-\log \left(1+\frac{X_{4}}{Y_{S G}}\right)
\end{aligned}
$$

where

$$
\begin{aligned}
X_{4} & =\frac{\left(x^{i}-\bar{x}^{i}\right)^{2}\left(y^{r}-\bar{y}^{r}\right)^{2}}{4(t-\bar{t})} \\
Y_{S K} & =(s-\bar{s})(t-\bar{t})(u-\bar{u})-\frac{1}{2}(u-\bar{u})\left(y^{r}-\bar{y}^{r}\right)^{2}-\frac{1}{2}(s-\bar{s})\left(x^{i}-\bar{x}^{i}\right)^{2}
\end{aligned}
$$

\footnotetext{
${ }^{3} Y_{S K}$ differs by a factor $-i$ from the Special geometry formula obtained from the prepotential in 4.1
} 
where here and in the following summation over repeated indices is understood. Therefore the correction to the scalar metric in the brane coordinates is:

$$
\partial_{p} \partial_{\bar{q}} \Delta K=\partial_{p} \partial_{\bar{q}} \log \left(1+\frac{X_{4}}{Y_{S G}}\right)
$$

It is clear that the classical brane coordinates are not good "supersymmetric" coordinates, in that the corrected action is not polynomial in them. From the fact that the combined system is a homogeneous space, we indeed expect that suitable coordinates exist such that the quantum corrected ( $N=2$ supersymmetric) action has a simple polynomial dependence on them, including the interference term. Such coordinates do indeed exist and allow to write the combined Born-Infeld action and supersymmetric counterterms, in a manifest supersymmetric way. Modulo field redefinitions, these coordinates reduce to the standard brane coordinates when either the $D 3$ or the $D 7$-branes are absent, in which cases the homogeneous space becomes a symmetric space. This parametrization in terms of "supersymmetric" coordinates, corresponds to the solvable Lie algebra description of the manifold which we shall discuss in what follows. In the previously introduced Alekseevski's notation the manifold under consideration is of type $K\left(n_{3}, n_{7}\right)$ which can be written as:

$$
\begin{aligned}
K\left(n_{3}, n_{7}\right) & =W\left(g_{\alpha}, h_{\alpha}, Y^{ \pm}, Z^{ \pm}\right) \\
\operatorname{dim}\left(Y^{ \pm}\right) & =n_{3} ; \operatorname{dim}\left(Z^{ \pm}\right)=n_{7}
\end{aligned}
$$

where $n_{3}$ and $n_{7}$ denote the number of $D 3$ and $D 7$-branes respectively. For the sake of clarity we rename in this section the generators $h_{1}, h_{2}, h_{3}$ and $g_{1}, g_{2}, g_{3}$ by $h_{t}, h_{u}, h_{s}$ and $g_{t}, g_{u}, g_{s}$ respectively. Our identification of the scalar fields with solvable parameters is described by the following expression for a generic solvable Lie algebra element:

$$
\begin{gathered}
\text { Solv }=\left\{\sum_{\alpha=t, u, s} \varphi^{\alpha} h_{\alpha}+\hat{\theta}_{t} g_{t}+\theta_{u} g_{u}+\theta_{s} g_{s}+y^{r \pm} Y_{r}^{ \pm}+z^{i \pm} Z_{i}^{ \pm}\right\} \\
\hat{\theta}_{t}=\theta_{t}+y^{r+} y^{r-}+z^{i+} z^{i-}
\end{gathered}
$$

where $\left(y^{r+}, y^{r-}\right)$ and $\left(z^{i+}, z^{i-}\right)$ are related to the real and imaginary parts of the $D 3$ and $D 7$-branes complex coordinates along $T^{2}$. The non trivial commutation relations between the above solvable generators are:

$$
\begin{aligned}
{\left[h_{t}, Y^{ \pm}\right] } & =\frac{1}{2} Y^{ \pm} ; \quad\left[h_{t}, Z^{ \pm}\right]=\frac{1}{2} Z^{ \pm} \\
{\left[h_{s}, Y^{ \pm}\right] } & = \pm \frac{1}{2} Y^{ \pm} ; \quad\left[h_{u}, Z^{ \pm}\right]= \pm \frac{1}{2} Z^{ \pm} \\
{\left[g_{s}, Y^{-}\right] } & =Y^{+} ; \quad\left[g_{u}, Z^{-}\right]=Z^{+} \\
{\left[Y_{r}^{+}, Y_{s}^{-}\right] } & =\delta_{r s} g_{t} ; \quad\left[Z_{i}^{+}, Z_{j}^{-}\right]=\delta_{i j} g_{t} ; r, s=1, \ldots, n_{3} i, j=1, \ldots, n_{7} \\
{\left[h_{\alpha}, g_{\alpha}\right] } & =g_{\alpha} ; \alpha=t, u, s
\end{aligned}
$$


We exponentiate the solvable algebra using the following coset-representative:

$$
L=e^{\theta_{s} g_{s}} e^{y^{r-} Y_{r}^{-}} e^{y^{r+} Y_{r}^{+}} e^{\theta_{u} g_{u}} e^{z^{i-} Z_{i}^{-}} e^{z^{i+} Z_{i}^{+}} e^{\hat{\theta}_{t} g_{t}} e^{\varphi^{\alpha} h_{\alpha}}
$$

The order of the exponentials in the coset representative and the particular parameter $\hat{\theta}_{t}$ used for $g_{t}$, have been chosen in such a way that the axions $\theta_{s}, \theta_{t}, \theta_{u}, y^{r+}, z^{i+}$ appear in the resulting metric only covered by derivatives. The metric reads:

$$
\begin{aligned}
d s^{2}= & \left(d \varphi_{\alpha}\right)^{2}+e^{-2 \varphi_{t}}\left(d \theta_{t}+\frac{1}{2} d \theta_{u}\left(z^{-}\right)^{2}+\frac{1}{2} d \theta_{s}\left(y^{-}\right)^{2}+z^{i-} d z^{i+}+y^{r-} d y^{r+}\right)^{2}+ \\
& e^{-2 \varphi_{u}} d \theta_{u}^{2}+e^{-2 \varphi_{s}} d \theta_{s}^{2}+e^{-\varphi_{t}-\varphi_{u}}\left(d z^{i+}+d \theta_{u} z^{i-}\right)^{2}+e^{-\varphi_{t}+\varphi_{u}}\left(d z^{i-}\right)^{2}+ \\
& e^{-\varphi_{t}-\varphi_{s}}\left(d y^{r+}+d \theta_{s} y^{r-}\right)^{2}+e^{-\varphi_{t}+\varphi_{s}}\left(d y^{r-}\right)^{2} \\
& \left(z^{+}\right)^{2} \equiv \sum_{i=1}^{n_{7}}\left(z^{i+}\right)^{2} ;\left(y^{+}\right)^{2} \equiv \sum_{r=1}^{n_{3}}\left(y^{r+}\right)^{2}
\end{aligned}
$$

Identifying the axionic coordinates $\theta_{s}, \theta_{t}, \theta_{u}, y^{r+}, z^{i+}$ with the real part of the special coordinates $s, t, u, y^{r}, x^{i}$, and comparing the corresponding components of the metric one easily obtains the following relations between the solvable coordinates and the special coordinates ${ }^{4}$ :

$$
\begin{aligned}
s & =\theta_{s}-\frac{i}{2} e^{\varphi_{s}} ; u=\theta_{u}-\frac{i}{2} e^{\varphi_{u}} \\
t & =\theta_{t}-\frac{i}{2}\left(e^{\varphi_{t}}+\frac{1}{2} e^{\varphi_{u}}\left(z^{-}\right)^{2}+\frac{1}{2} e^{\varphi_{s}}\left(y^{-}\right)^{2}\right) \\
x^{i} & =z^{i+}+\frac{i}{2} e^{\varphi_{u}} z^{i-} ; y^{r}=y^{r+}+\frac{i}{2} e^{\varphi_{s}} y^{r-}
\end{aligned}
$$

Let us notice that the classical B-I+C-S action (4.2), with no interference term in the D3 $(c, d)$ and $D 7(a, b)$ brane coordinates is still described by a homogeneous manifold spanned by the following $2 n_{3}+2 n_{7}+6$ isometries:

$$
\begin{aligned}
u & \rightarrow e^{\lambda_{u}} u ; \delta u=u_{0}+a_{0}^{i} b^{i} \\
s & \rightarrow e^{\lambda_{s}} s ; \delta s=s_{0}+c_{0}^{r} d_{r} \\
t & \rightarrow e^{\lambda_{t}} t ; \delta t=t_{0} \\
c^{r} & \rightarrow e^{\frac{\lambda_{s}+\lambda_{t}}{2}} c^{r} ; \delta c^{r}=t_{0} d^{r} \\
d^{r} & \rightarrow e^{\frac{\lambda_{s}-\lambda_{t}}{2}} d^{r} ; \delta d^{r}=d_{0}^{r} \\
a^{i} & \rightarrow e^{\frac{\lambda_{u}+\lambda_{t}}{2}} a^{i} ; \delta a^{i}=a_{0}^{i}+t_{0} b^{i} \\
b^{i} & \rightarrow e^{\frac{\lambda_{u}-\lambda_{t}}{2}} b^{i} ; \delta b^{i}=b_{0}^{i} .
\end{aligned}
$$

\footnotetext{
${ }^{4}$ We notice that in the previous paper [24] the imaginary parts of $u$ and $t$ were chosen to be positive. This however is inconsistent with the positivity domain of the vector kinetic terms which requires $s, t, u$ to have negative imaginary parts. Indeed $\Im s$ and $\Im u$ appear as coefficients in the kinetic terms of the $D 7$ and D3-brane vectors.
} 
The underlying homogeneous space is generated by the following rank 3 solvable Lie algebra $\left\{T_{a}^{i}, T_{b}^{i}, T_{c}^{r}, T_{d}^{r}, h_{s}, h_{t}, h_{u}, g_{s}, g_{t}, g_{u}\right\}$ whose non trivial commutation relations are:

$$
\begin{aligned}
{\left[T_{a}^{i}, T_{b}^{j}\right] } & =\delta^{i j} g_{u} ; \quad\left[T_{c}^{r}, T_{d}^{s}\right]=\delta^{r s} g_{s} \\
{\left[T_{b}^{i}, g_{t}\right] } & =T_{a}^{i} ; \quad\left[T_{d}^{r}, g_{t}\right]=T_{c}^{r} \\
{\left[h_{\alpha}, g_{\alpha}\right] } & =g_{\alpha} \quad \alpha=s, t, u \\
{\left[h_{s}, T_{d}^{r}\right] } & =\frac{1}{2} T_{d}^{r} ; \quad\left[h_{s}, T_{c}^{r}\right]=\frac{1}{2} T_{c}^{r} \\
{\left[h_{u}, T_{b}^{i}\right] } & =\frac{1}{2} T_{b}^{i} ; \quad\left[h_{u}, T_{a}^{i}\right]=\frac{1}{2} T_{a}^{i} \\
{\left[h_{t}, T_{d}^{r}\right] } & =-\frac{1}{2} T_{d}^{r} ; \quad\left[h_{t}, T_{c}^{r}\right]=\frac{1}{2} T_{c}^{r} \\
{\left[h_{t}, T_{b}^{i}\right] } & =-\frac{1}{2} T_{b}^{i} ; \quad\left[h_{t}, T_{a}^{i}\right]=\frac{1}{2} T_{a}^{i},
\end{aligned}
$$

where the nilpotent generators have been labelled by the corresponding axionic scalar fields. This space is not a subspace of the original quanternionic space, but it becomes so if we set either $a, b=0$ and exchange the role of $s$ and $t$ or if we set $c, d=0$ and exchange the role of $u$ and $t$.

The amazing story is that the coordinates in $D=4$ corresponding to the supersymmetric theory, deform this space into an other homogeneous space generated by the isometries in (4.8) which corresponds to an $N=2$ Special Geometry.

The relation between the solvable Lie algebra generators $\left\{T_{a}^{i}, T_{b}^{i}, T_{c}^{r}, T_{d}^{r}, h_{s}, h_{t}, h_{u}, g_{s}, g_{t}, g_{u}\right\}$ corresponding to the classical coordinates and the solvable generators $\left\{Y^{ \pm}, Z^{ \pm}, h_{\alpha}, g_{\alpha}\right\}$ corresponding to the "supersymmetric" coordinates is the following:

$$
\begin{aligned}
T_{a}^{i} & =\hat{Z}^{i+} ; T_{b}^{i}=\hat{Z}^{i-} \\
T_{c}^{r} & =\hat{Y}^{r+} ; T_{d}^{r}=\hat{Y}^{r-}
\end{aligned}
$$

where $\hat{Y}$ and $\hat{Z}$ are the generators with opposite grading with respect to $Y$ and $Z$ respectively. It can be shown that in the manifold $K\left(n_{3}, n_{7}\right), \hat{Y}$ or $\hat{Z}$ are isometries only if $n_{7}=0$ or $n_{3}=0$ respectively. Indeed in these two cases the manifold is symmetric and each solvable nilpotent isometry has a "hidden" counterpart with opposite grading. Otherwise the manifold spanned by the classical coordinates and the manifold parametrized by the "supersymmetric" ones are in general different. 


\section{Discussion on the $D=6$ dimensional origin of the prepotential}

The cubic prepotential for the $D 3, D 7$ branes can be obtained in a number of ways by using different string dualities.

Type I theory, obtained as a Type IIB orientifold on $K 3$, in the presence of $D 5$ and $D 9$ branes has an effective description in terms of an $N=1$ theory in $D=6[$ [ ] . In this theory there are $n_{t}$ tensor multiplets and $n_{v}$ vector multiplets $\left(n_{t}=1\right.$ in models equivalent to perturbative heterotic string). Upon further compactification on $S^{1}$ to $D=5$, one obtains a Very Special geometry of the type [7]

$$
\begin{aligned}
\mathcal{F}= & z b^{r} \eta_{r s} b^{s}-b^{r} C_{r x y} a^{x} a^{y} \\
& x, y=1, \ldots, n_{v} ; r, s=1, \ldots, n_{t}+1,
\end{aligned}
$$

where the physical scalar fields are $n_{t}+n_{v}+1$, of which $n_{t}$, described by components of $b_{r}$, come from the tensor multiplets in $D=6, n_{v}$, namely $a^{x}$, from the $D=6$ vectors and one coincides with the Kaluza-Klein scalar $z\left(\eta_{r s}\right.$ is the lorentzian metric with signature $\left(1, n_{t}\right)$ of the tensor multiplets). For $n_{t}=1$

$$
\mathcal{F}=z b c-b v_{x y} a^{x} a^{y}-c \tilde{v}_{x y} a^{x} a^{y}
$$

and we obtain the Very Special Geometry described in section 4. This result for $n_{t}=1$ is "dual" to heterotic string on $K 3 \times S^{1}$ or M-theory on a Calabi-Yau threefold which is an elliptic fibration [7]. The case $n_{t}>1$ corresponds in heterotic theory to non-perturbative vacua.

In the Type I setting the special geometry is seen to arise from a combined Born-Infeld Lagrangian and Chern-Simons couplings, with the addition of suitable supersymmetry anomaly counterterms. However in the $D=5$ context the special geometry is simply dictated by the Chern-Simons five-dimensional coupling

$$
d_{A B C} \int A^{A} \wedge F^{B} \wedge F^{C},
$$

which specifically gives the following terms [7]

$$
Z d B d C ; C v_{x y} d A^{x} d A^{y} ; B \tilde{v}_{x y} d A^{x} d A^{y},
$$

which determine uniquely the prepotential.

For the case $n_{t}=1$ the prepotential corresponds to a homogeneous symmetric space [24]. Inspection of the prepotential for $n_{t}>1$ indicates that the space is homogeneous when the 
coefficients $C_{r x y}$ satisfy some Clifford algebra relations and some further relations between $n_{t}$ and $n_{v}$ hold true [21, 22, 23. In this respect it would be interesting to see whether some superstring models with $n_{t}>1$ exist with a homogeneous special geometry, as in the $n_{t}=1$ case.

\section{Acknowledgements}

We would like to thank C. Angelantonj for enlightening discussions. R.D. and M.T. would like to thank the Physics Department of CERN, where part of this work was done, for its kind hospitality. S.F. would like to thank the Physics Department of Politecnico di Torino where part of this work was done, for its kind hospitality.

The work of S.F. has been supported in part by the D.O.E. grant DE-FG03-91ER40662, Task C, and in part by the European Community's Human Potential Program under contract HPRN-CT-2000-00131 Quantum Space-Time, in association with INFN Frascati National Laboratories and R.D. and M. T. are associated to Torino University.

\section{A Appendix.}

In this appendix we compute the explicit expression of the Riemann tensor for the dual Kähler manifold as defined in [6] and discussed in section 3 .

We start from a very Special Kähler manifold of complex dimension $n$, characterized by a cubic prepotential:

$$
F(X)=\frac{1}{X^{0}} d_{a b c} X^{a} X^{b} X^{c} ; a, b, c=1, \ldots, n
$$

We denote be $\lambda^{a}=\Im z^{a}$, $z^{a}$ corresponding to the special coordiate parametrization of the space. It is useful to introduce the following quantities:

$$
\kappa(\lambda)=d_{a b c} \lambda^{a} \lambda^{b} \lambda^{c} ; \quad \kappa_{a}(\lambda)=d_{a b c} \lambda^{b} \lambda^{c} ; \quad \kappa_{a b}(\lambda)=d_{a b c} \lambda^{c} ; \quad V(\lambda)=\frac{\kappa(\lambda)}{6}
$$

The Kähler potential is:

$$
K(\lambda)=-\log (V(\lambda))
$$

The metric of the manifold and its inverse are therefore:

$$
\begin{aligned}
G_{a \bar{b}} & =\frac{\partial^{2} K}{\partial \lambda^{a} \partial \lambda^{b}}=-6\left(\frac{\kappa_{a b}}{\kappa}-\frac{3}{2} \frac{\kappa_{a} \kappa_{b}}{\kappa^{2}}\right) \\
\left(G^{-1}\right)^{a \bar{b}} & =-\frac{1}{6}\left(\left(\kappa^{-1}\right)^{a b} \kappa-3 \lambda^{a} \lambda^{b}\right)
\end{aligned}
$$


The connection and the Riemann tensor are:

$$
\begin{aligned}
\Gamma_{a b}^{c}= & \left(G^{-1}\right)^{c \bar{c}} \partial_{a} G_{\bar{c} b}=-i\left(d_{a b \bar{c}}\left(\kappa^{-1}\right)^{c \bar{c}}-6 \frac{\delta_{(a}^{c} \kappa_{b)}}{\kappa}+3 \frac{\kappa_{a b} \lambda^{c}}{\kappa}\right) \\
R_{a b}{ }^{d c}= & -\left(G^{-1}\right)^{d \bar{d}} \partial_{\bar{d}} \Gamma_{a b}^{c}=-i\left(G^{-1}\right)^{d f} \frac{\partial}{\partial \lambda^{f}} \Gamma_{a b}^{c}= \\
& -2 \delta_{(a}^{c} \delta_{b)}^{d}+d_{a b f}\left(\kappa^{-1}\right)^{f(c} \lambda^{d)}+\frac{1}{2}\left(\kappa^{-1}\right)^{c d} \kappa_{a b}-\frac{\kappa}{6}\left(\kappa^{-1}\right)^{d \bar{d}}\left(\kappa^{-1}\right)^{c e}\left(\kappa^{-1}\right)^{\bar{c} f} d_{\bar{d} e f} d_{a b \bar{c}}
\end{aligned}
$$

I can be easily verified that the above expression coincides with the general formula for the Riemann tensor in the Special Geometry, namely

$$
R_{a b}^{d{ }^{c}}=-2 \delta_{(a}^{c} \delta_{b)}^{d}+e^{2 K} C_{a b m} C^{c d m}
$$

if we identify $C_{a b c}$ with $d_{a b c}$.

We now define the "dual" Kähler manifold by the following transformation of the imaginary parts of the complex coordinates: $t_{a}=\kappa_{a} / 2$. The dual metric is

$$
\begin{aligned}
g^{a \bar{b}} & =\frac{36}{\kappa^{2}}\left(G^{-1}\right)^{a \bar{b}}=\frac{\partial^{2} \hat{K}}{\partial t_{a} \partial t_{b}} \\
\hat{K} & =2 K
\end{aligned}
$$

The connection and the Riemann tensor for the dual manifold are:

$$
\begin{aligned}
\tilde{\Gamma}_{d}^{b c}= & \frac{6}{\kappa}\left(G^{-1}\right)^{c q} \Gamma_{q d}^{b}=i\left(d_{q d f}\left(\kappa^{-1}\right)^{c q}\left(\kappa^{-1}\right)^{b f}+\frac{6}{\kappa} \delta_{d}^{(b} \lambda^{c)}-\frac{3}{\kappa}\left(\kappa^{-1}\right)^{c b} \kappa_{d}\right) \\
\tilde{R}_{a d d}^{c b}= & -i\left(g^{-1}\right)_{a f} \frac{\partial}{\partial t^{f}} \tilde{\Gamma}_{d}^{b c}= \\
& -\delta_{(a}^{c} \delta_{d)}^{b}-d_{f e(a} \kappa_{d)}\left(\kappa^{-1}\right)^{c f}\left(\kappa^{-1}\right)^{b e}+\left(\kappa^{-1}\right)^{c b} \kappa_{a d}+\frac{\kappa}{3}\left(\kappa^{-1}\right)^{e(c}\left(\kappa^{-1}\right)^{b) f}\left(\kappa^{-1}\right)^{q p} d_{e p a} d_{f q d}
\end{aligned}
$$

We have verified, by means of computer aided computations, that, for all homogeneous symmetric $N=2$ manifolds

$$
R_{a b}{ }^{d}{ }^{c}=\tilde{R}_{a b}{ }^{d}{ }^{c}
$$

which signals that the dual manifolds are of the same kind. Aside from the homogeneous symmetric manifolds we have considered an instance of non-homogeneous manifold defined by

$$
V(\lambda)=d_{1}\left(\lambda^{1}\right)^{3}+d_{2}\left(\lambda^{2}\right)^{3}
$$

and an instance of homogeneous non-symmetric manifold, namely $K(1,1)$. In both cases equality (A.9) does not hold and therefore the two spaces are necessarily different. 


\section{References}

[1] S. Cecotti, S. Ferrara and L. Girardello, "Geometry Of Type II Superstrings and The Moduli of Superconformal Field Theories," Int. J. Mod. Phys. A 4 (1989) 2475.

[2] S. Ferrara and S. Sabharwal, "Quaternionic Manifolds for Type II Superstring Vacua of Calabi-Yau Spaces," Nucl. Phys. B 332 (1990) 317.

[3] R. Bohm, H. Gunther, C. Herrmann and J. Louis, "Compactification of type IIB string theory on Calabi-Yau threefolds," Nucl. Phys. B 569 (2000) 229 arXiv:hep-th/9908007.

[4] K. Becker, M. Becker, M. Haack and J. Louis, "Supersymmetry breaking and alpha'corrections to flux induced potentials," JHEP 0206 (2002) 060 arXiv:hep-th/0204254.

[5] M. Grana, T. W. Grimm, H. Jockers and J. Louis, "Soft supersymmetry breaking in Calabi-Yau orientifolds with D-branes and fluxes," arXiv:hep-th/0312232; T. W. Grimm and J. Louis, "The effective action of $\mathrm{N}=1$ Calabi-Yau orientifolds," arXiv:hep-th/0403067.

[6] R. D'Auria, S. Ferrara and M. Trigiante, "c-map, Very Special quaternionic geometry and dual Kaehler spaces," arXiv:hep-th/0401161.

[7] S. Ferrara, R. Minasian and A. Sagnotti, "Low-Energy Analysis of $M$ and $F$ Theories on Calabi-Yau Threefolds," Nucl. Phys. B 474 (1996) 323 arXiv:hep-th/9604097.

[8] I. Antoniadis, C. Bachas, C. Fabre, H. Partouche and T. R. Taylor, "Aspects of type I - type II - Heterotic Triality in Four Dimensions," Nucl. Phys. B 489 (1997) 160 arXiv:hep-th/9608012.

[9] R. Kallosh and S. Prokushkin, "Supercosmology," arXiv:hep-th/0403060.

[10] T. Watari and T. Yanagida, "GUT phase transition and hybrid inflation," arXiv:hep-ph/0402125.

[11] A. Linde, "Prospects of inflation," arXiv:hep-th/0402051

[12] J. P. Hsu and R. Kallosh, "Volume stabilization and the origin of the inflaton shift symmetry in string theory," arXiv:hep-th/0402047.

[13] S. Kachru, M. B. Schulz and S. Trivedi, "Moduli stabilization from fluxes in a simple IIB orientifold," JHEP 0310 (2003) 007 arXiv:hep-th/0201028. 
[14] A. R. Frey and J. Polchinski, "N = 3 warped compactifications," Phys. Rev. D 65 (2002) 126009 arXiv:hep-th/0201029.

[15] R. D'Auria, S. Ferrara and S. Vaulá, " $\mathrm{N}=4$ gauged supergravity and a IIB orientifold with fluxes," New J. Phys. 4 (2002) 71 arXiv:hep-th/0206241; R. D'Auria, S. Ferrara, M. A. Lledo and S. Vaulá, "No-scale $N=4$ supergravity coupled to YangMills: The scalar potential and super Higgs effect," Phys. Lett. B 557 (2003) 278 arXiv:hep-th/0211027; R. D'Auria, S. Ferrara, F. Gargiulo, M. Trigiante and S. Vaulá, " $\mathrm{N}=4$ supergravity Lagrangian for type IIB on $\mathrm{T}^{* *} 6 / \mathrm{Z}(2)$ in presence of fluxes and D3-branes," JHEP 0306 (2003) 045 arXiv:hep-th/0303049.

[16] L. Andrianopoli, R. D'Auria, S. Ferrara, P. Fré and M. Trigiante, "R-R scalars, U-duality and solvable Lie algebras," Nucl. Phys. B 496 (1997) 617 arXiv:hep-th/9611014; L. Andrianopoli, R. D’Auria, S. Ferrara, P. Fré, R. Minasian and M. Trigiante, "Solvable Lie algebras in Type IIA, Type IIB and M theories," Nucl. Phys. B 493 (1997) 249 arXiv:hep-th/9612202; M. Trigiante, "Dualities in supergravity and solvable Lie algebras," arXiv:hep-th/9801144 M. Bertolini and M. Trigiante, "Regular R-R and NS-NS BPS black holes," Int. J. Mod. Phys. A 15 (2000) 5017 arXiv:hep-th/9910237.

[17] B. de Wit, H. Samtleben and M. Trigiante, "Maximal supergravity from IIB flux compactifications," Phys. Lett. B 583 (2004) 338 arXiv:hep-th/0311224.

[18] C. Angelantonj, S. Ferrara and M. Trigiante, "New D = 4 gauged supergravities from $\mathrm{N}=4$ orientifolds with fluxes," JHEP 0310 (2003) 015 arXiv:hep-th/0306185.

[19] C. Angelantonj, S. Ferrara and M. Trigiante, "Unusual gauged supergravities from Type IIA and Type IIB orientifolds," arXiv:hep-th/0310136

[20] D. V. Alekseevskii, "Classification of quaternionic spaces with a transitive solvable group of motions", Izv. Akad. Nauk SSSR Ser. Mat. 9, 315-362 (1975); Math. USSR Izvesstija, 9, 297-339 (1975).

[21] S. Cecotti, "Homogeneous Kahler Manifolds And T Algebras in N=2 Supergravity and Superstrings," Commun. Math. Phys. 124 (1989) 23.

[22] B. de Wit and A. Van Proeyen, "Symmetries of Dual Quaternionic Manifolds," Phys. Lett. B 252 (1990) 221.

[23] B. de Wit, F. Vanderseypen and A. Van Proeyen, "Symmetry structure of special geometries," Nucl. Phys. B 400 (1993) 463 arXiv:hep-th/9210068. 
[24] C. Angelantonj, R. D'Auria, S. Ferrara and M. Trigiante, " $K 3 \times T^{2} / \mathbb{Z}_{2}$ orientifolds with fluxes, open string moduli and critical points," Phys. Lett. B 583 (2004) 331 arXiv:hep-th/0312019.

[25] E. Cremmer and A. Van Proeyen, "Classification of Kahler Manifolds in N=2 Vector Multiplet Supergravity Couplings," Class. Quant. Grav. 2 (1985) 445.

[26] A. Giryavets, S. Kachru, P. K. Tripathy and S. P. Trivedi, "Flux compactifications on Calabi-Yau threefolds," arXiv:hep-th/0312104.

[27] L. Andrianopoli, R. D'Auria and S. Ferrara, "Consistent reduction of $\mathrm{N}=2 \rightarrow \mathrm{N}=$ 1 four dimensional supergravity coupled to matter," Nucl. Phys. B 628 (2002) 387 arXiv:hep-th/0112192. 